1 Relation of seasonal birth pulses and maternal immunity with viral invasion and persistence: A

2 case study of Hendra virus infection in a population of black flying foxes (Pteropus alecto)

4 Jaewoon JEONG ${ }^{1,2 *}$, Alison J. PEEL ${ }^{1}$, Raina K. PLOWRIGHT ${ }^{3}$, Olivier RESTIF ${ }^{4}$, and Hamish

$5 \quad$ McCALLUM $^{1}$

$7 \quad{ }^{1}$ Environmental Futures Research Institute, Griffith University, Brisbane, Queensland 4111,

8 Australia

$9 \quad{ }^{2}$ Department of Health Management, Atlantic Veterinary College, University of Prince Edward

10 Island, Charlottetown, Prince Edward Island C1A 4P3, Canada

11 Department of Microbiology and Immunology, Montana State University, Bozeman, Montana

12 59717, U.S.A

$13{ }^{4}$ Disease Dynamics Unit, Department of Veterinary Medicine, University of Cambridge,

14 Cambridge, UK

15

16 Corresponding author: Jaewoon Jeong (jjeong@upei.ca)

17 Current address: Atlantic Veterinary College, University of Prince Edward Island, 550

18 University Avenue, Charlottetown, Prince Edward Island, Canada, C1A 4P3

19 Phone: +1-902-566-0579, Fax: +1-902-620-5053

20 Running head: Effects of maternal immunity on epidemics in seasonally breeding bats 
21 Abstract

22 Increasing outbreaks of emerging infectious diseases, originating from wildlife, has

23 intensified interest in understanding the dynamics of these diseases in their wildlife reservoir

24 hosts. Until recently, the effect of seasonal birth pulses and subsequent waning of maternally

25 derived antibodies on epidemics in a wild mammal population has received little attention and

26 has remained obscure. In this study, we explore how population structure, influenced by seasonal

27 breeding and maternally derived immunity, affects viral invasion and persistence, using a

28 hypothetical system loosely based on Hendra virus infection in black flying foxes (Pteropus

29 alecto). We used deterministic epidemic models to simulate transient epidemics, following viral

30 introduction into an infection-free population, with a variety of timings within a year and

31 different levels of pre-existing herd immunity. Moreover, we applied different levels of birth

32 synchrony and different modelling methods of waning maternal immunity to examine the effect

33 of birth pulses and maternally derived immunity, both individually and in combination. The

34 presence of waning maternal immunity dispersed the supply time of susceptible individuals in

35 seasonally breeding populations, hence diminishing the effect of birth pulse. Dampened

36 epidemics, caused by waning maternal immunity, made viral invasion and persistence easier.

37 This study enhanced our understanding of viral invasion, persistence, and timing of epidemics in

38 wildlife populations.

40 Keywords: seasonal birth, maternal immunity, population dynamics, Hendra virus, black flying

41 foxes 
44 Introduction

45 Seasonal behaviour of wildlife hosts - including aggregation, reproduction, migration, and

46 associated physiological variations - influences the transmission of infection in their populations

47 [1]. Among the seasonal factors, breeding has been implicated as playing a role in determining

48 pathogen persistence and epidemic patterns. Seasonal births result in seasonal variation in

49 population size and proportion of susceptible hosts, affecting infection of the population [2].

50 Seasonal breeding could result in a population requiring a much larger size to maintain a

51 pathogen [3]. The frequency of birth pulses within a year has also been suggested as a major

52 factor determining viral persistence [4]. Moreover, seasonal breeding can drive the timing of

53 recurrent epidemics in a wildlife population [5].

Births from susceptible mothers and immigration of susceptible individuals are not the

55 only sources of virus-susceptible individuals. While newborns of immune mothers can obtain

56 protection against infection via maternally-derived antibodies (MatAb), this protection wanes

57 over a certain period [6], causing these individuals to enter the susceptible pool as juveniles. The

58 proportion of immune mothers that confer waning passive immunity to newborns could,

59 therefore, significantly modify the supply of susceptible hosts. The supply of susceptible hosts

60 from these two sources (from births to susceptible mothers and via loss of MatAb [7]) interact

61 with each other to determine whether viral introduction into a population would result in viral

62 invasion or persistence. The presence of MatAb in seasonally breeding wildlife is known to

63 delay recurrent epidemics over a multi-year timescale [8]. However, the mechanism underlying

64 the effect of this delay on the timing of epidemics within a year requires further investigation. 
A modelling approach was established to describe the infectious period by an exponential

66 distribution, which is a classical method to transfer individuals across infection stages [9] and is

67 implicit in a simple differential equation SIR (susceptible-infectious-immune) model with

68 constant rate parameters [10]. Although the exponential distribution has been used widely for

69 computational ease, efforts towards finding more realistic methods to model the infectious period

70 resulted in the development of gamma-distributed infectious periods [11]. Wearing, Rohani (12)

71 addressed the significance of modelling the infectious and latency periods in predicting the

72 impact of infectious diseases. Although maternal immune period has been modelled using a

73 conventional exponential distribution, for both infectious and latency periods, the effect of this

74 method on the model outcomes has been poorly studied. Therefore, it is worthwhile to explore

75 the mechanism of generation of different epidemic modelling outcomes by an exponential and a

76 gamma distribution. This would, in turn, help to determine whether the method of modelling

77 waning maternal immunity is an important influence on model behaviour.

Bats (Order: Chiroptera) have been identified as natural reservoirs of many emerging

79 infectious diseases of public health concern $[13,14]$. They do not appear to suffer from many of

80 these infections, especially those caused by viruses, despite the obvious exceptions of rabies and

81 Tacaribe virus $[15,16]$. However, the fatality rates attributable to bat viral diseases are often

82 quite high in other mammalian species [13]. For example, Hendra virus has been detected in four

83 species of flying foxes (Pteropus alecto, P. conspicillatus, P. poliocephalus, and P. scapulatus)

84 in Australia [17]. . Spillover from P. alecto and P. conspicillatus [18-20] to horses, and thereafter

85 to humans, causes serious clinical symptoms and even death in both horses and humans [21].

86 Excretion of Hendra virus by flying foxes rarely results in spillover events; excreted virus has to

87 overcome several barriers such as pathogen survival and spread in the environment, and 
88 domestic animal or human exposure $[22,23]$. Surveillance of Hendra virus spillover events has

89 revealed temporal patterns in the southern subtropics of eastern Australia between June and

90 September [24]. Seasonal behaviour of reservoir hosts, such as seasonal breeding, needs to be

91 investigated to examine how it can influence the seasonal characteristics of viral prevalence [3,

92 25]. Although seasonal factors such as birth pulses are not the only drivers of Hendra virus

93 dynamics, and other factors (Eucalyptus phenology, bat movement, temperature, and nutrition)

94 can also significantly influence Hendra virus spillover [22, 26], it would be valuable to

95 understand the underlying mechanism of this influence, since Hendra virus spillover is known to

96 result from a variety of processes with many interacting factors involved [23].

97 Using a system loosely based on Hendra virus infection in a population of P. alecto, we

98 investigate the effect of seasonal birth pulses and waning MatAb on viral invasion and

99 persistence in a certain population. Moreover, we explore the hypothesis that seasonal birth

100 pulses and waning MatAb can facilitate the seasonal clustering of Hendra virus epidemics in $P$.

101 alecto. We deterministically model a hypothetical population of $P$. alecto with two varying

102 conditions of herd immunity (proportion of recovered individuals in the population at the time of

103 viral introduction) and days from birth pulse to viral introduction (days from the last birth pulse

104 before viral introduction until viral introduction) in every model.

106 Methods

107 Model structure

108 We simulated the Hendra virus dynamics in $P$. alecto using compartmental deterministic

109 models, which were framed using ordinary differential equations (ODEs) and numerically 
110 integrated using the deSolve package [27] in R [28]. Profiles of Hendra virus prevalence and

111 seroprevalence in flying foxes may be generated by at least three different underlying

112 mechanisms: susceptible-infectious-recovered (SIR), susceptible-infectious-recovered-

113 susceptible (SIRS), and susceptible-infectious-latent-infectious (SILI) [29]. Among the three

114 scenarios, we use assume the SIR here, but recognize that other disease processes, such as SILI,

115 are highly likely. The purpose of this study was to explore the effect of maternally derived

116 immunity on viral prevalence; therefore, factors such as loss of acquired immunity and latent

117 infection were excluded in our models to increase modelling transparency and to ease the

118 interpretation of modelling results. In addition to the SIR model, an MSIR model was built by

119 adding a maternally immune (M) compartment to the existing SIR model. Maternally immune

120 (M) newborns become susceptible at the rate $\delta$ (Table 1). Susceptible (S) bats become infected at

121 the rate $\beta$ SI (assuming density-dependent transmission). Bats are infectious (I) before recovery at

122 the rate $\gamma$. Based on the SIR model, we assumed that once recovered (R), bats remain immune for

123 the rest of their life (modelled in [30] along with the scenario of latency and reactivation).

124 Hendra virus infection is not known to not cause clinical disease in its reservoir hosts (flying

125 foxes) [31]; therefore, infected bats are assumed to have the same mortality rate as susceptible

126 and recovered bats. The models were simulated with annual birth pulses for eight years and run

127 with a daily time step.

128 Table 1. Model parameters.

\begin{tabular}{lllll}
\hline \hline Parameter & Symbol & Value & Unit & Reference \\
\hline Transmission rate & $\beta$ & 0.0000476 & $\begin{array}{l}\text { Per capita per } \\
\text { day }\end{array}$ \\
Recovery rate & $\gamma$ & $1 / 7$ & $\begin{array}{l}\text { Per capita per } \\
\text { day }\end{array}$
\end{tabular}




\begin{tabular}{|c|c|c|c|c|}
\hline Mortality rate & $\mu$ & $1 / 7$ & $\begin{array}{l}\text { Per capita per } \\
\text { year }\end{array}$ & {$[32]$} \\
\hline $\begin{array}{l}\text { Maternally-derived immunity losing } \\
\text { rate }\end{array}$ & $\delta$ & $1 / 255$ & $\begin{array}{l}\text { Per capita per } \\
\text { day }\end{array}$ & {$[33]$} \\
\hline Ageing rate & $\alpha$ & $1 / 2$ & $\begin{array}{l}\text { Per capita per } \\
\text { year }\end{array}$ & {$[34]$} \\
\hline Scalar to control birth rate & $\kappa$ & 0.00159 & $\begin{array}{l}\text { Per adult per } \\
\text { day }\end{array}$ & $\begin{array}{l}\text { This } \\
\text { study }\end{array}$ \\
\hline $\begin{array}{l}\text { Low, medium and high birth pulse } \\
\text { synchronies }\end{array}$ & s & $\begin{array}{l}0.013,1.3, \text { and } \\
130\end{array}$ & - & {$[3]$} \\
\hline Timing of birth pulse & $\varphi$ & $1 / 2$ & - & {$[32]$} \\
\hline Initial herd immunity & & $0.55 \sim 0.85$ & - & \\
\hline $\begin{array}{l}\text { Days from the last birth pulse to viral } \\
\text { introduction }\end{array}$ & & $0 \sim 365$ & day & \\
\hline Colony size & $\mathrm{N}$ & 10000 & capita & {$[30]$} \\
\hline $\begin{array}{l}\text { Average proportion of juvenile bats } \\
\text { in total bats }\end{array}$ & $\eta$ & 0.23 & - & {$[32]$} \\
\hline Gamma-distribution parameter & $\mathrm{g}$ & 8 & & {$[33]$} \\
\hline
\end{tabular}

Simulation of seasonal birth pulses and maternally-derived immunity required an age-

131 structured model of bat population. The age-structure consisted of sexually immature juvenile

132 bats (denoted by the subscript $i$ ) and sexually mature adult bats (subscript $m$ ). In the MSIR

133 model, juveniles became adults, which could breed two years after their birth (at the rate $\varepsilon$ ) [34].

134 The proportion of juveniles in the total population $(\eta)$ was, on average, 0.24 [32]. Four epidemic

135 compartments for the two age groups led to a total of eight stages (see Supplementary Equations

136 S1 and S2). Although maternally immune adults are not expected to exist in nature, we included

137 this stage for modelling consistency. The exponential distribution of periods of maternally-

138 derived immunity means that some individuals stay in the maternally immune compartment

139 longer than in any period between birth and adulthood. With the parameters used, only few 
maternally immune adults remained, and hence had an insignificant effect on the results. We

141 assumed an age-independent annual mortality rate $(\mu)$ of $16 \%$ [32]. Mortality rate $(\mu)$ and birth

142 rate were independent of population density and were so chosen that the population size

143 remained constant. Bats born to immune female adults $\left(R_{m}\right)$ and to maternally immune female

144 adults $\left(M_{m}\right)$ were assumed to be maternally immune $\left(M_{i}\right)$ in the MSIR model, whereas all

145 newborns were assumed to be susceptible $\left(S_{i}\right)$ in the SIR model. The initial numbers in each

146 compartment are described in Supplementary Text S1 and Supplementary Table S1.

147 Seasonally pulsed births were modelled with a periodic Gaussian function (PGF) [3, 4]: b(t)

$148=\kappa \sqrt{\mathrm{S} / \pi} \mathrm{e}^{-\operatorname{scos}^{2}(\pi \mathrm{t}-\varphi)}$, where $\kappa$ controls the magnitude, $\mathrm{s}$ determines synchrony, and $\varphi$

149 determines the timing of birth pulse. This function allows births to occur exclusively in a certain

150 period within a year, with none outside this period $[3,4]$. The scaling parameter $(\kappa)$ was used so

151 that the total population size would be stable inter-annually. $P$. alecto shows different seasonal

152 birth patterns, depending on latitude [32]. In southeast Queensland and Northern NSW, where

153 most Hendra virus spillover events have occurred till date, the offspring of $P$. alecto are known

154 to be born in October and November, which is closely aligned to that of P. poliocephalus [32,

155 35]. With reference to the seasonal birth pulse of $P$. poliocephalus, we set $\mathrm{s}=130$, so that $95 \%$

156 of annual births are concentrated within a one-month period [3] (see Supplementary Fig. S1 for

157 annual fluctuation of the number of individuals). In addition to high levels of synchrony ( $\mathrm{s}=$

158 130), we also modelled low $(\mathrm{s}=0.013)$ and medium $(\mathrm{s}=1.3)$ levels of birth synchrony for

159 comparison.

160 Flying fox colonies are patchily distributed, and individuals move among colonies. This

161 movement may introduce Hendra virus into infection-free colonies, which may thereafter trigger

162 transient epidemics (causing metapopulation dynamics of the virus within the host populations 
163 [30]). As the natural reservoir hosts of Hendra virus, flying fox colonies are highly likely to have

164 been previously exposed to the virus [30], and so colonies are not necessarily immunologically

165 naïve at the timing of viral introduction. Partial immunity of a population affects the duration and

166 size of an epidemic caused by the viral introduction [36]. We therefore designed models to

167 simulate Hendra virus dynamics in a colony of flying foxes within this broader context of a

168 metapopulation in eastern Australia, assuming that a proportion of bats in a colony had

169 previously been exposed to Hendra virus, and hence, was partially immune.

Although the transmission mode of Hendra virus among flying foxes is uncertain, a

171 combination of frequency and density dependence has been hypothesised [24]. This study

172 assumed density-dependent transmission, affected by population size, rather than frequency-

173 dependent transmission, which remains unaffected by population size. Serological surveys of

174 wild flying foxes showed an average proportion of susceptible individuals of $60 \%[37,38]$, and

175 by with this finding, Plowright, Foley (30) estimated the mean values of a Hendra virus

176 transmission rate $(\beta)$ of 0.0000476 and recovery rate $(\gamma)$ of $1 / 7$ days $^{-1}$, which we used in our

177 models (Table 1$)$. The loss rate of maternally derived immunity $(\delta)$ was assumed as $1 / 255$ day $^{-1}$,

178 based on the data from an earlier experimental study on eight newborn P. alecto (born to

179 seropositive bats) [33].

Gamma-distributed periods of maternally-derived immunity

The MSIR model with exponentially distributed waning periods of MatAb (hereafter

182 referred to as exponential MSIR model) assumed that the number of maternally immune hosts

183 decreased exponentially since birth. In addition to the exponential MSIR model, an MSIR model

184 with gamma-distributed waning periods of MatAb (hereafter referred to as gamma MSIR model)

185 was simulated. This model used a gamma distribution in transferring maternally immune 
186 juveniles to susceptible juveniles. Gamma-distributed waning periods of MatAb were modelled

187 by dividing the maternally immune stage into multiple (g) sub-stages, where gamma-distributed

188 waning periods of MatAb had a mean of $g$ and variance of 1/g [12] (see Supplementary Equation

$189 \mathrm{~S} 3$ ). As the gamma distribution parameter (g) increased, the rate of loss of maternally immune

190 individuals shifted from an exponential decay to a fixed duration of maternal immunity. Epstein,

191 Baker (33) determined the mean duration of maternal immunity (255 days) and half-lives for

192 serum antibody against Hendra virus in twelve pups of $P$. alecto. Using the half-lives, we

193 calculated the duration of maternal immunity in each pup so that the mean duration would be 255

194 days. We then modelled the gamma-distributed maternally immune durations with a variety of

195 gamma distribution parameters and found that the mean duration was the most closely achieved

196 with $\mathrm{g}=8$. Thus, we used the gamma distribution parameter $(\mathrm{g}=8)$ for our models

197 (Supplementary Fig. S2). This study included nine models: low, medium, and high levels of birth

198 synchrony $(\mathrm{s}=0.013,1.3$, and 130) in each of the three different modelling methods in terms of

199 waning of MatAb: SIR model, exponential MSIR model, and gamma MSIR model.

Pseudo-extinction

Viral persistence, which is an essentially stochastic process, should normally be analysed

202 using stochastic models [39]. Nevertheless, deterministic models with a cut-off to determine viral

203 persistence may provide valuable insights. We used pseudo-extinction, in which the number of

204 infectious bats dropping below a cut-off was assumed to be a proxy for viral extinction in a

205 population. If the sum of infectious juveniles $\left(I_{i}\right)$ and infectious adults $\left(I_{m}\right)$ dropped below one,

206 in the population we assumed that the infection failed to persist, and hence, the simulations were

207 stopped. We applied varying conditions of herd immunity and days from birth pulse to viral

208 introduction, since herd immunity could be an important factor in determining the magnitude 
209 and duration of an epidemic [30] and days from birth pulse to viral introduction might affect

210 viral persistence in seasonally breeding wildlife [3]. We used deterministic models over

211 stochastic models, since the former were faster so that it was appropriate to use when we

212 simulate with a wide ranges of herd immunity and timing of viral introduction. Since viral

213 extinction is likely to occur in the first few post-epidemic troughs [40], the simulation period was

214 limited to six years, which was considered to be long enough to include the first few epidemics

215 and the following troughs.

217 Results

218 Epidemics, caused by the introduction of an infectious bat into a bat population having a

219 wide range of immunities, but no current infections, were simulated with the SIR model,

220 exponential MSIR model, and gamma MSIR model. The predominant effect of herd immunity

221 on epidemics, compared to the effect of birth synchrony and maternally-derived immunity, made

222 the difference of epidemics insignificant in all three models at herd immunity $<0.55$. However,

223 differences occurred when herd immunity was similar to the herd immunity of an endemic

224 equilibrium state (approximately between 0.55 and 0.7 ).

\section{Viral invasion}

227 time over low birth synchrony (s=1.3) (Error! Reference source not found.). With high birth

228 synchrony $(\mathrm{s}=130)$, epidemics occurred at a broader range and higher levels of herd immunity. In

229 particular, viral introduction shortly before a birth pulse (Fig. 1, DB >300), was found to result in

230 the highest magnitude of epidemics. Compared to the SIR model, the addition of MatAb in the 
231 exponential MSIR model resulted in more uniform steady magnitude of epidemics across the

232 timings of viral introduction at high birth synchrony $(\mathrm{s}=130)$, but higher maximum epidemic

233 magnitudes at low and medium birth pulse synchrony ( $\mathrm{s}=13)$. Compared to the exponential

234 MSIR model, the gamma MSIR model showed even more uniform epidemic magnitudes across

235 the timings of viral introduction (at high birth synchrony), with little difference observed at low

236 and medium synchrony. Waning MatAb therefore appeared to reduce the effect of a highly

237 seasonal birth pulse by dispersing the timing of supply of susceptible individuals, and this effect

238 was most pronounced in the gamma MSIR model compared to the exponential MSIR.

239 Fig. 1. Magnitude of epidemics. The colour key shows the maximum number of infected

240 individuals in the epidemic following viral introduction into a population. Herd immunity

241 indicates the proportion of recovered individuals in a population, and days from birth pulse to

242 viral introduction refers to the days of viral introduction since the peak of the previous birth

243 pulse. Top, middle, and bottom panels show the results from SIR, exponential MSIR, and

244 gamma MSIR models, respectively. Left, centre, and right panels show the results from low,

245 medium, and high birth synchrony $(\mathrm{s}=0.013,1.3$, and 130$)$, respectively. White parts indicate no

246 epidemic.

247 Viral fadeout and persistence

We examined the effect of herd immunity and number of days from birth pulse to viral

249 introduction on viral persistence. Introduction of infected individuals did not result in

250 maintenance of infections in the population when birth synchrony was low ( $\mathrm{s}=1.3)$ or when

251 synchrony was high (s=130) but there was no MatAb (Error! Reference source not found.).

252 Medium birth synchrony ( $\mathrm{s}=13$ ) appeared optimal for viral persistence. With high birth

253 synchrony, if viral introduction occurred in presence of many susceptible individuals, very large

254 epidemics were followed by deep troughs. Otherwise, if viral introduction occurred in presence

255 of only few susceptible individuals, epidemics were not triggered, let alone viral persistence. The

256 mechanisms of viral fadeout were described in Supplementary Text S2. 
Fig. 2. Number of infected individuals at the troughs following epidemics. Layout of panels is explained in Figure 1, but the results from low birth synchrony are not shown here. White parts indicate viral extinction.

Regarding viral persistence, the highest number of infected individuals at the troughs,

261 following epidemic peaks, was found in two parts of the parameter space, for both small and

262 large values of days from birth pulse to viral introduction ( $a$ and $b$ in the middle panel of the

263 right column in Error! Reference source not found.). The part 'a' was induced when a birth

264 pulse correlated with decreasing prevalence, shortly after viral introduction; the part ' $b$ ' arose

265 when a birth pulse correlated with decreasing prevalence after epidemic peaks. Compared to the

266 exponential MSIR model, the gamma MSIR model showed more combinations of days from

267 birth pulse to viral introduction and herd immunity that allowed viral persistence with high birth

268 synchrony ( $\mathrm{s}=130)$. This effect arose because gamma-distributed periods of maternally derived

269 immunity resulted in the relatively steady supply of susceptible hosts throughout the year, just as

270 medium birth synchrony $(\mathrm{s}=13)$ resulted in a steadier supply of susceptible hosts compared to

271 high birth synchrony. Thus, the steady supply caused less intense epidemics and less deep

272 (shallow) troughs.

273 Timing of epidemic peaks

274 Overall, maternally-derived immunity delayed the timing of epidemic peaks. Maternally-

275 derived immunity dispersed the supply of susceptible bats into the population, and thereby the

276 number of infected bats relatively gradually increased, developing over a longer time period

277 before peaking. When the timing of epidemic peaks was displayed in terms of months within a

278 year, the SIR model showed seasonally clustered epidemic peaks, although this feature was

279 weakened in the exponential MSIR model, and hardly observed in the gamma MSIR model (Fig.

280 3). When herd immunity was similar to herd immunity of an endemic equilibrium state in SIR 
model, the epidemic peaks mostly appeared within one year after viral introduction in the SIR model with birth pulses. However, in the exponential MSIR model, the epidemic peaks were delayed compared to those in the SIR model and showed a broader spectrum of timings than in the SIR model (Supplementary Fig. S3). In the gamma MSIR model, after viral introduction, the epidemics required a specific period to reach their peak, and the periods until epidemic peaks were delayed compared to those in the exponential MSIR model. key shows the month when the epidemics reach their peaks following viral introduction into a population. Layout of panels is explained in Fig. 1. MSIR model, we modelled the number of maternally immune bats to decrease by as much as $1 / 255$ of the remaining maternally immune bats every day. As a result, most juvenile bats lost their maternal immunity soon after birth, rather than 255 days after birth (Supplementary Fig. S2). When herd immunity was high and maternally immune newborns were more common than susceptible newborns, the temporal trend of epidemics was expected to change noticeably at 255

298 (see Supplementary Fig. S4 for the number of individuals in each compartment across time).

299 However, the number of infectious individuals began to rise at the time of birth pulses, which

300 could probably be attributed to the exponential method of modelling the loss of maternally

301 derived immunity. In comparison, the gamma MSIR model showed a more enhanced effect of

302 maternally derived immunity (Error! Reference source not found.). In the gamma MSIR

303 model, loss of maternally derived immunity seemed to occur mainly at 255 days after birth.

304 Therefore, the impact of maternally derived immunity in determining epidemic pattern was much 
305 higher than in the exponential MSIR model. Moreover, the two relatively separate timings

306 ensured a steady supply of susceptible individuals throughout the year, decreasing the extent of

307 seasonal clustering of epidemic peaks. Another reason for birth pulses having a stronger impact

308 on epidemics than did loss of maternally derived immunity was that the temporal synchrony of

309 birth pulses was higher than that of loss of maternally derived immunity. Although more

310 susceptible hosts were supplied from the loss of maternally derived immunity than from birth

311 pulses, the tighter span of the latter relative to the former had a high impact on epidemic patterns.

312 Figure 4. Supply of susceptible individuals from birth pulses and waning maternal immunity.

313 Layout of panels is explained in Figure 1. The number of days since the last birth pulse before

314 viral introduction until the viral introduction into a colony was 10, and herd immunity of the

315 population was 0.7 .

317 Discussion

This study suggests that the effect of maternally-derived immunity in seasonal breeding

319 species is not negligible and should be considered to improve our understanding of viral invasion

320 and persistence in wildlife host populations. Modelling results showed that loss of maternally

321 derived immunity disperses the timing of supply of susceptible individuals. The dispersed timing

322 causes an increase in the likelihood of viral persistence and shifts the timing of epidemic peaks

323 farther away from the timing of a birth pulse. However, these conclusions need to be interpreted

324 with caution, since the modelling results are contingent on the assumptions made. In particular,

325 SIR dynamics is one of at least three possible scenarios that describes Hendra virus dynamics

326 [29]. Therefore, prediction of the timing of Hendra virus excretion pulses cannot be achieved by

327 the results obtained from our models; instead, this study identified the relative importance of 
328 births and waning maternally derived antibody as the sources of susceptible hosts, and the

329 significance of modelling methods of waning maternally derived antibody.

330 Seasonal clustering of Hendra virus spillover has been observed only in southeast

331 Queensland and Northern NSW, whereas it occurred in northeast Queensland throughout the

332 year [24]. While P. alecto in southeast Queensland and Northern NSW has a high birth

333 synchrony, that in a more northern area has an inconsistent birth synchrony [32]. The modelling

334 results showed medium birth synchrony to result in less temporal clustering of epidemic peaks

335 than high birth synchrony (Fig. 3). The different temporal trend of spillover could, therefore, be

336 associated with different synchrony of birth pulses of $P$. alecto based on latitude. Other climatic

337 or ecological factors may also contribute to the different timing of spillover events (Peel et al.

338 2017) [23].

As flying foxes are natural reservoir hosts of Hendra virus [41], the virus must be

340 maintained within the flying fox populations. However, seasonal birth pulses make it more

341 difficult for the virus to persist compared to a population that has a constant birth rate [1].

342 Furthermore, when the annual birth pulse is tight, the disadvantage is further intensified [3]. For

343 seasonally breeding flying foxes, other mechanisms are required to overcome or offset the

344 disadvantageous conditions for viral persistence. Our modelling results support the implication

345 that MatAb can play a role in mitigating adverse conditions for the maintenance of non-

346 pathogenic virus, by dispersing the timing of supply of susceptible hosts into the population

347 [42]. This is consistent with recent stochastic models showing that MatAb supports the

348 maintenance of a henipavirus and lyssavirus in seasonally breeding African fruit bats (Eidolon

349 helvum) $[7,42]$. 
Wearing, Rohani (12) demonstrated that infectious disease modelling-based predictions

351 are significantly affected by whether the infectious and latent periods are modelled using an

352 exponential distribution or a gamma distribution. Heterogeneity in the longevity of protective

353 immunity in different individuals requires mechanisms to deal with the distribution of immune

354 periods of individuals in a model structure [43]. Here, we showed that gamma-distributed

355 periods of maternally-derived immunity could generate significantly different modelling results

356 compared to exponentially distributed periods. Since the functional form of the loss of MatAb

357 against Hendra virus or against other viruses is unknown, we set $\mathrm{g}=8$ to illustrate the effect of

358 reducing the coefficient of variation in the duration of immunity by a third, namely from $100 \%$

359 (exponential distribution) to about 35\% $(1 / \sqrt{g})$ ), trying to realistically imitate waning MatAb.

360 Results of the gamma MSIR model should not be interpreted as demonstrating a more accurate

361 prediction than that by the exponential MSIR model; it merely shows that substantially different

362 modelling results can be generated depending on whether the loss of maternally-derived

363 immunity is exponentially or gamma-distributed. Therefore, appropriate modelling of when

364 maternally immune newborns lose their passive immunity, depending on species and pathogens

365 against emerging infectious diseases is expected to help improve the prediction of disease

366 outbreaks.

Although this study modelled epidemics in a single population, the results obtained should

368 be considered in the context of metapopulation structure, since this study assumed SIR dynamics

369 in which metapopulations would be expected to play an important role in Hendra virus

370 maintenance $[29,30]$. An effect of the seasonality of epidemics in metapopulations is that

371 seasonal forcing of transmission may cause the epidemics in each population to be synchronised

372 [44]. Synchronised epidemics are likely to increase the probability of viral fadeout in a 
373 metapopulation. Viral introduction would be less probable if epidemics in populations become

374 extinct at similar times. It is, therefore, necessary to examine the effects of seasonality of viruses

375 on the synchrony of epidemics in the metapopulation models of hosts.

376 Given the growing interest in emerging zoonotic diseases from wildlife [45], there is a

377 need to combine empirical studies of zoonotic reservoir ecology with mathematical models to

378 develop a mechanistic understanding of virus persistence and spillover. By incorporating

379 maternally derived immunity into generic epidemic models, we have provided a framework to

380 study epidemics in seasonally breeding wildlife species. Complex effects of demographic and

381 virus-related parameters have previously been reported in other systems [5]. We explored a range

382 of plausible assumptions to complement the limited empirical data available for the dynamics of

383 Hendra virus in flying fox populations, and the results showed the effects of maternally derived

384 immunity on the timing of epidemic peaks, rather than predicting the actual timing of epidemic

385 peaks. Caution must be exercised, however, when arriving at conclusions concerning the effect

386 of maternally derived immunity, given the specific circumstances associated with each disease

387 and host population structure.

SUPPORTING INFORMATION

390 Additional Supporting Information may be found in the online version of this article: 
393 We thank J. McBroom, L. Grogan, D. Kerlin, K. Wells, and J. Giles for assistance in analysing

394 data and structuring models. We thank Stephanie Palmer for editing this manuscript.

396 Financial support

397 This work was supported by the Commonwealth of Australia, the states of New South Wales and

398 Queensland under the National Hendra Virus Research Program, awarded through the Rural

399 Industries Research and Development Corporation (RIRDC). JJ was supported by Griffith

400 University Postgraduate Research Scholarship and Griffith University International Postgraduate

401 Research Scholarship and was supported by Ocean Frontier Institute. AJP was supported by a

402 Queensland Government Accelerate Postdoctoral Research Fellowship. RKP, AJP, OR, and HM

403 were supported by DARPA BAAHR001118S0017 D18AC00031 and the National Science

404 Foundation DEB-1716698. RKP was supported by funds from DARPA D16AP00113, the

405 National Institute of General Medical Sciences of the National Institutes of Health under Award

406 Number P20GM103474 and P30GM110732, and SERDP RC-2633. OR acknowledges funding

407 from the ALBORADA Trust.

408

409 Declaration of interest

410 None

411

412 References 
413 1. Altizer S, Dobson A, Hosseini P, Hudson P, Pascual M, Rohani P. Seasonality and the

414 dynamics of infectious diseases. Ecology Letters. 2006;9(4):467-84. PubMed PMID:

415 WOS:000236384100011.

$416 \quad 2 . \quad$ Altizer S, Hochachka WM, Dhondt AA. Seasonal dynamics of mycoplasmal

417 conjunctivitis in eastern North American house finches. Journal of Animal Ecology.

418 2004;73(2):309-22. PubMed PMID: WOS:000189258400010.

$4193 . \quad$ Peel AJ, Pulliam JRC, Luis AD, Plowright RK, O'Shea TJ, Hayman DTS, et al. The

420 effect of seasonal birth pulses on pathogen persistence in wild mammal populations. Proceedings

421 of the Royal Society B-Biological Sciences. 2014;281(1786). doi: ARTN 20132962

422 10.1098/rspb.2013.2962. PubMed PMID: WOS:000336784500005.

423 4. Hayman DT. Biannual birth pulses allow filoviruses to persist in bat populations.

424 Proceedings of the Royal Society of London B: Biological Sciences. 2015;282(1803):20142591.

425 5. Begon M, Telfer S, Smith MJ, Burthe S, Paterson S, Lambin X. Seasonal host dynamics

426 drive the timing of recurrent epidemics in a wildlife population. Proceedings of the Royal

427 Society B-Biological Sciences. 2009;276(1662):1603-10. PubMed PMID:

428 WOS:000264445000008.

429 6. Hasselquist D, Nilsson J-Å. Maternal transfer of antibodies in vertebrates: trans-

430 generational effects on offspring immunity. Philosophical Transactions of the Royal Society of

431 London B: Biological Sciences. 2009;364(1513):51-60.

432 7. Hayman DT, Luis AD, Restif O, Baker KS, Fooks AR, Leach C, et al. Maternal antibody

433 and the maintenance of a lyssavirus in populations of seasonally breeding African bats. PloS one.

434 2018;13(6):e0198563.

435 8. Garnier R, Gandon S, Harding KC, Boulinier T. Length of intervals between epidemics:

436 evaluating the influence of maternal transfer of immunity. Ecology and Evolution.

437 2014;4(5):568-75. PubMed PMID: WOS:000332139800003.

4389 9. Anderson RM, May RM. Infectious diseases of humans : dynamics and control. Oxford:

439 Oxford University Press; 1991. viii, 757 p. p.

440 10. Keeling MJ, Rohani P. Modeling infectious diseases in humans and animals. Princeton:

441 Princeton University Press; 2008. xi, 366 p. p.

442 11. Lloyd AL. Realistic distributions of infectious periods in epidemic models: changing

443 patterns of persistence and dynamics. Theoretical population biology. 2001;60(1):59-71.

444 12. Wearing HJ, Rohani P, Keeling MJ. Appropriate models for the management of

445 infectious diseases (vol 2, pg e174, 2005). Plos Medicine. 2005;2(8):813-. doi: ARTN e320

446 10.1371/journal.pmed.0020320. PubMed PMID: WOS:000231676900036.

447 13. Calisher CH, Childs JE, Field HE, Holmes KV, Schountz T. Bats: Important reservoir

448 hosts of emerging viruses. Clinical Microbiology Reviews. 2006;19(3):531-+. doi:

449 10.1128/Cmr.00017-06. PubMed PMID: WOS:000239419600004.

450 14. Luis AD, Hayman DTS, O'Shea TJ, Cryan PM, Gilbert AT, Pulliam JRC, et al. A

451 comparison of bats and rodents as reservoirs of zoonotic viruses: are bats special? Proceedings of

452 the Royal Society B-Biological Sciences. 2013;280(1756). PubMed PMID:

453 WOS:000315461500012.

454 15. Turmelle A, Jackson F, Green D, McCracken G, Rupprecht C. Host immunity to repeated

455 rabies virus infection in big brown bats. Journal of General Virology. 2010;91(9):2360-6.

456 16. Brook CE, Dobson AP. Bats as 'special'reservoirs for emerging zoonotic pathogens.

457 Trends in microbiology. 2015;23(3):172-80. 
17. Halpin K, Young PL, Field HE, Mackenzie JS. Isolation of Hendra virus from pteropid bats: a natural reservoir of Hendra virus. Journal of General Virology. 2000;81:1927-32. PubMed PMID: WOS:000088509900005. Hendra Virus Excretion in Naturally-Infected Flying-Foxes: Implications for Viral Transmission and Spillover Risk. Plos One. 2015;10(10). PubMed PMID: WOS:000363184600092. Infection Dynamics in the Grey-Headed Flying Fox (Pteropus poliocephalus) at the SouthernMost Extent of Its Range: Further Evidence This Species Does Not Readily Transmit the Virus to Horses. Plos One. 2016;11(6). PubMed PMID: WOS:000377824800006.

20. Field H, Jordan D, Edson D, Morris S, Melville D, Parry-Jones K, et al. Spatiotemporal Aspects of Hendra Virus Infection in Pteropid Bats (Flying-Foxes) in Eastern Australia. Plos One. 2015;10(12). PubMed PMID: WOS:000365891600087.

21. Field HE, Breed AC, Shield J, Hedlefs RM, Pittard K, Pott B, et al. Epidemiological perspectives on Hendra virus infection in horses and flying foxes. Australian Veterinary Journal. 2007;85(7):268-70. doi: 10.1111/j.1751-0813.2007.00170.x. PubMed PMID: WOS:000247941200021. 22. Paez DJ, Giles J, Mccallum H, Field H, Jordan D, Peel A, et al. Conditions affecting the timing and magnitude of Hendra virus shedding across pteropodid bat populations in Australia. Epidemiology \& Infection. 2017;145(15):3143-53.

23. Plowright RK, Parrish CR, McCallum H, Hudson PJ, Ko AI, Graham AL, et al. Pathways to zoonotic spillover. Nature Reviews Microbiology. 2017.

480 24. Plowright RK, Eby P, Hudson PJ, Smith IL, Westcott D, Bryden WL, et al. Ecological dynamics of emerging bat virus spillover. Proceedings of the Royal Society B-Biological Sciences. 2015;282(1798). doi: ARTN 20142124

10.1098/rspb.2014.2124. PubMed PMID: WOS:000344595000009.

25. Baker KS, Suu-Ire R, Barr J, Hayman DT, Broder CC, Horton DL, et al. Viral antibody dynamics in a chiropteran host. Journal of animal ecology. 2014;83(2):415-28.

26. Martin G, Yanez-Arenas C, Plowright RK, Chen C, Roberts B, Skerratt LF. Hendra Virus Spillover is a Bimodal System Driven by Climatic Factors. EcoHealth. 2018:1-17.

490 28. R Core Team. R: A language and environment for statistical computing. R Foundation for Statistical Computing, Vienna, Austria. URL https://www.R-project.org/. 2016. 29. Plowright RK, Peel AJ, Streicker DG, Gilbert AT, McCallum H, Wood J, et al. Transmission or Within-Host Dynamics Driving Pulses of Zoonotic Viruses in Reservoir-Host Populations. Plos Neglected Tropical Diseases. 2016;10(8). doi: ARTN e0004796 10.1371/journal.pntd.0004796. PubMed PMID: WOS:000382390800005. 30. Plowright RK, Foley P, Field HE, Dobson AP, Foley JE, Eby P, et al. Urban habituation, ecological connectivity and epidemic dampening: the emergence of Hendra virus from flying foxes (Pteropus spp.). Proceedings of the Royal Society B-Biological Sciences. 2011;278(1725):3703-12. doi: 10.1098/rspb.2011.0522. PubMed PMID:

501 31. Halpin K, Young P, Mackenzie JS. Bats and viruses-a long association. American Journal 502 of Tropical Medicine \& Hygiene. 2001;65:249-. 
32. McIlwee A, Martin L. On the intrinsic capacity for increase of Australian flying-foxes (Pteropus spp., Megachiroptera). Australian Zoologist. 2002;32(1):76-100. 33. Epstein JH, Baker ML, Zambrana-Torrelio C, Middleton D, Barr JA, DuBovi E, et al. Duration of Maternal Antibodies against Canine Distemper Virus and Hendra Virus in Pteropid Bats. Plos One. 2013;8(6). PubMed PMID: WOS:000321150000090. Hendra Virus Persistence in Australian Flying-Fox Populations. Plos One. 2013;8(11). doi:

$510 \quad$ ARTN e80430

512 35. Vardon MJ, Tidemann CR. Reproduction, growth and maturity in the black flying-fox, Pteropus alecto (Megachiroptera : Pteropodidae). Australian Journal of Zoology. 1998;46(4):329-44. PubMed PMID: WOS:000078490800002. 36. Pulliam JRC, Dushoff JG, Levin SA, Dobson AP. Epidemic Enhancement in Partially Immune Populations. Plos One. 2007;2(1). PubMed PMID: WOS:000207444000015. 37. Plowright RK, Field HE, Smith C, Divljan A, Palmer C, Tabor G, et al. Reproduction and nutritional stress are risk factors for Hendra virus infection in little red flying foxes (Pteropus scapulatus). Proceedings of the Royal Society B-Biological Sciences. 2008;275(1636):861-9. doi: 10.1098/rspb.2007.1260. PubMed PMID: WOS:000253166300016.

38. Breed AC, Breed MF, Meers J, Field HE. Evidence of Endemic Hendra Virus Infection in Flying-Foxes (Pteropus conspicillatus)-Implications for Disease Risk Management. Plos One. 2011;6(12). doi: ARTN e28816

39. Gilligan CA, van den Bosch F. Epidemiological models for invasion and persistence of pathogens. Annual Review of Phytopathology. 2008;46:385-418. PubMed PMID: WOS:000259199300016.

40. King AA, Shrestha S, Harvill ET, Bjornstad ON. Evolution of Acute Infections and the Invasion-Persistence Trade-Off. American Naturalist. 2009;173(4):446-55. PubMed PMID: WOS:000263937300005.

41. Halpin K, Young P, Field H, Mackenzie J. Isolation of Hendra virus from pteropid bats: a natural reservoir of Hendra virus. Journal of General Virology. 2000;81(8):1927-32.

42. Peel AJ, Baker KS, Hayman DT, Broder CC, Cunningham AA, Fooks AR, et al. Support for viral persistence in bats from age-specific serology and models of maternal immunity. Scientific reports. 2018;8(1):3859.

43. Antia A, Ahmed H, Handel A, Carlson NE, Amanna IJ, Antia R, et al. Heterogeneity and longevity of antibody memory to viruses and vaccines. PLoS biology. 2018;16(8):e2006601.

44. Grassly NC, Fraser C. Seasonal infectious disease epidemiology. Proceedings of the

Royal Society of London B: Biological Sciences. 2006;273(1600):2541-50.

540 45. Rhyan J, Spraker T. Emergence of diseases from wildlife reservoirs. Veterinary 541 pathology. 2010;47(1):34-9. 


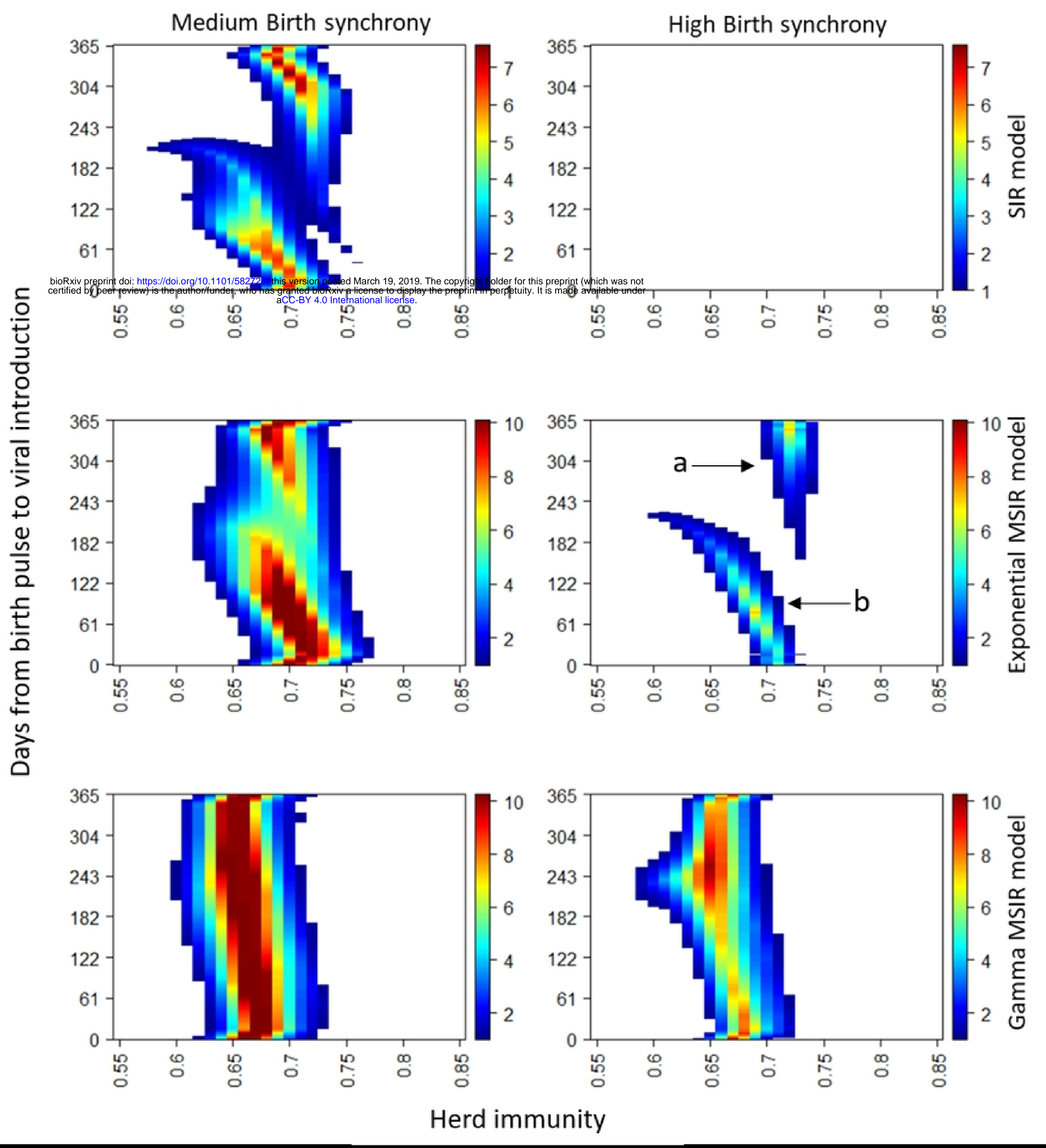

Fig. 2 
Low Birth synchrony

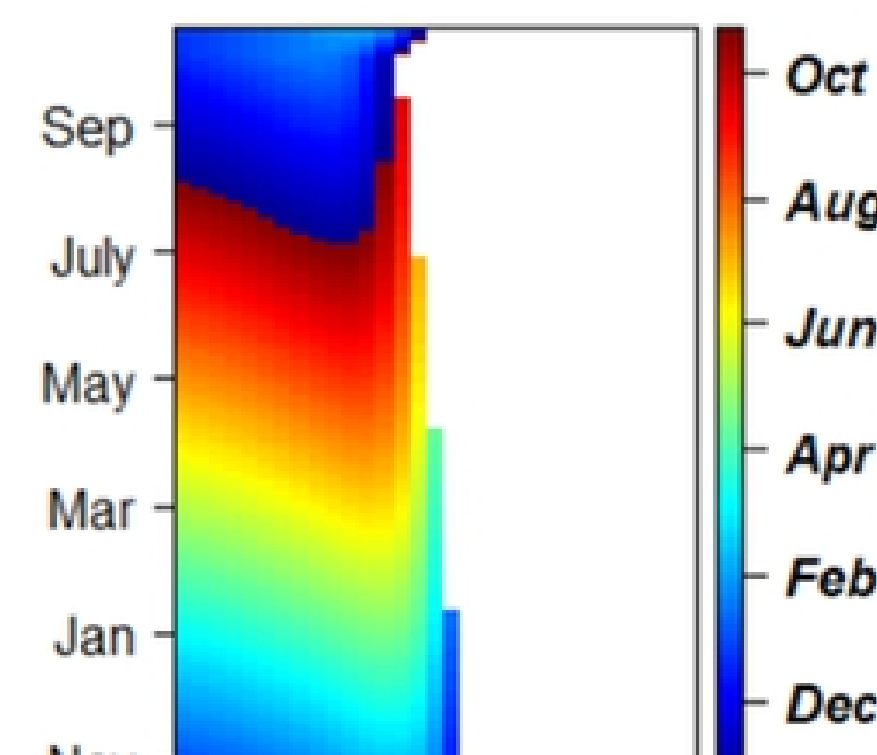

Medium Birth synchrony

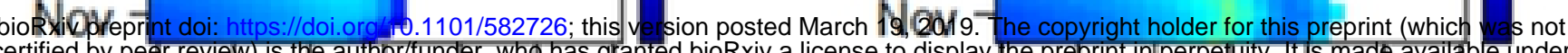

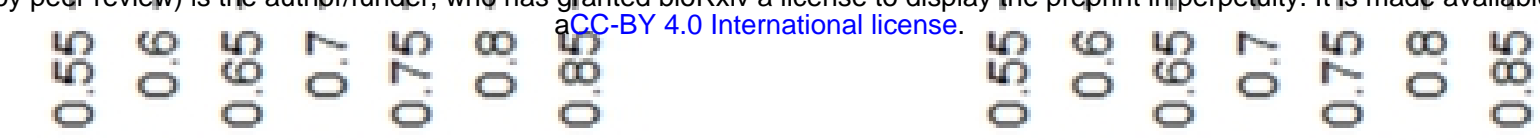
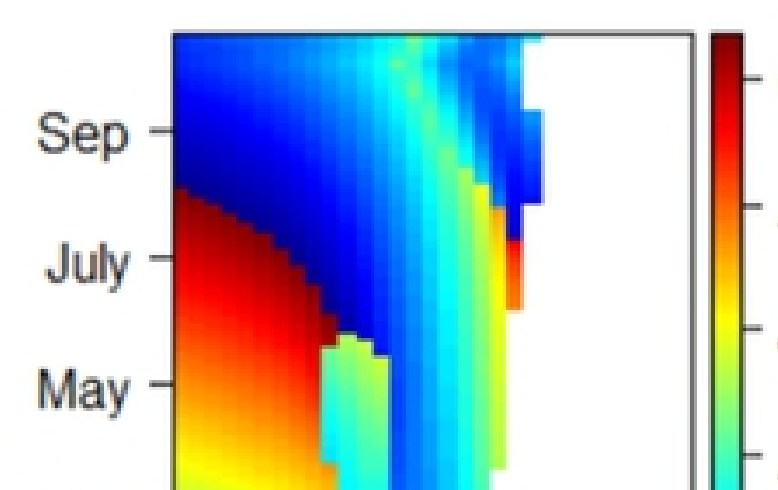

Mar -
Jan -

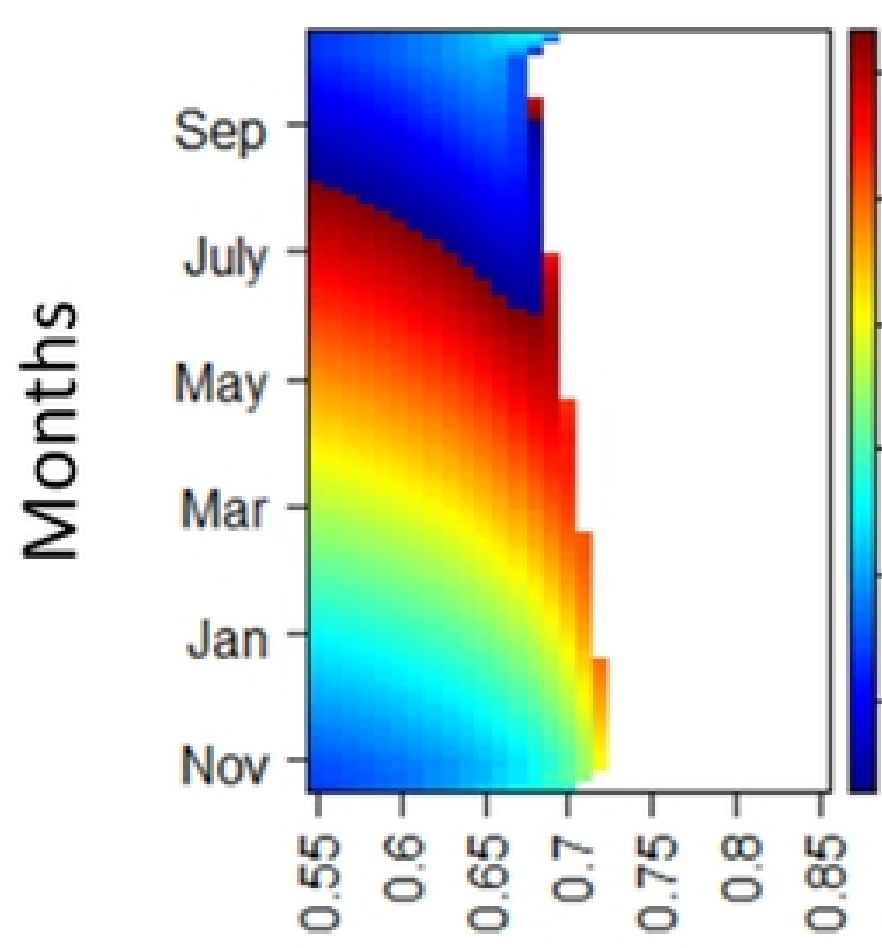

Oct
-Aug
- Jun
$-A p r$
- Feb
-Dec

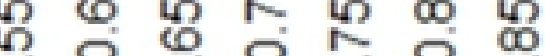

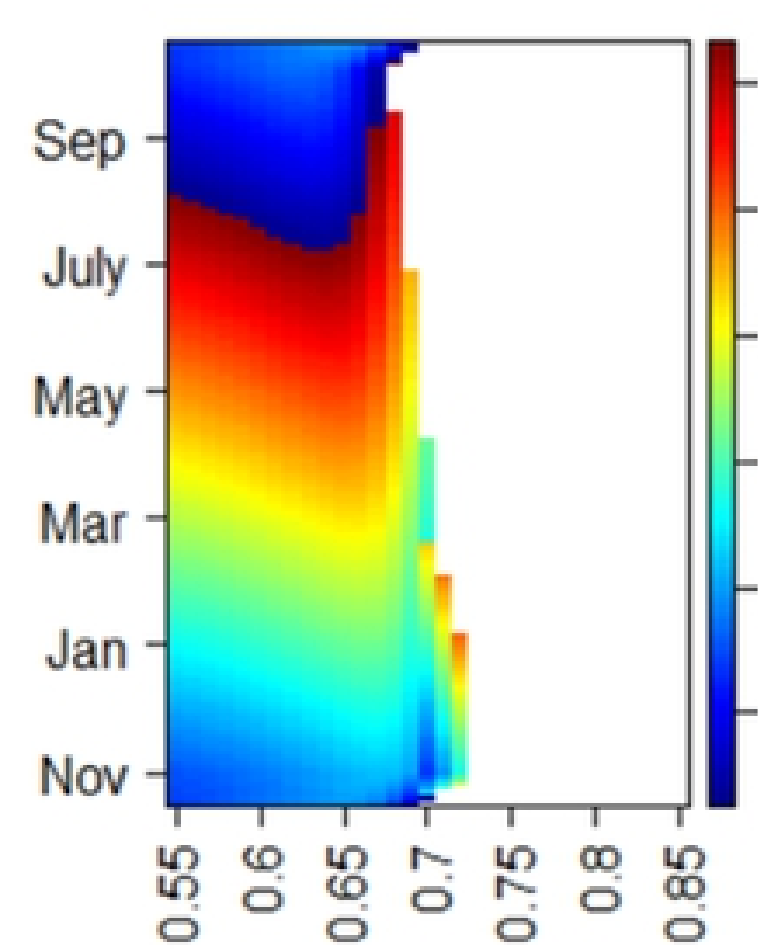

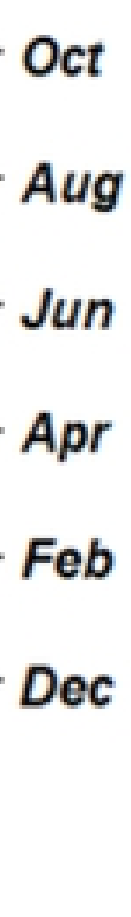

oct

Aug

Jun

Apr

Dec
Feb

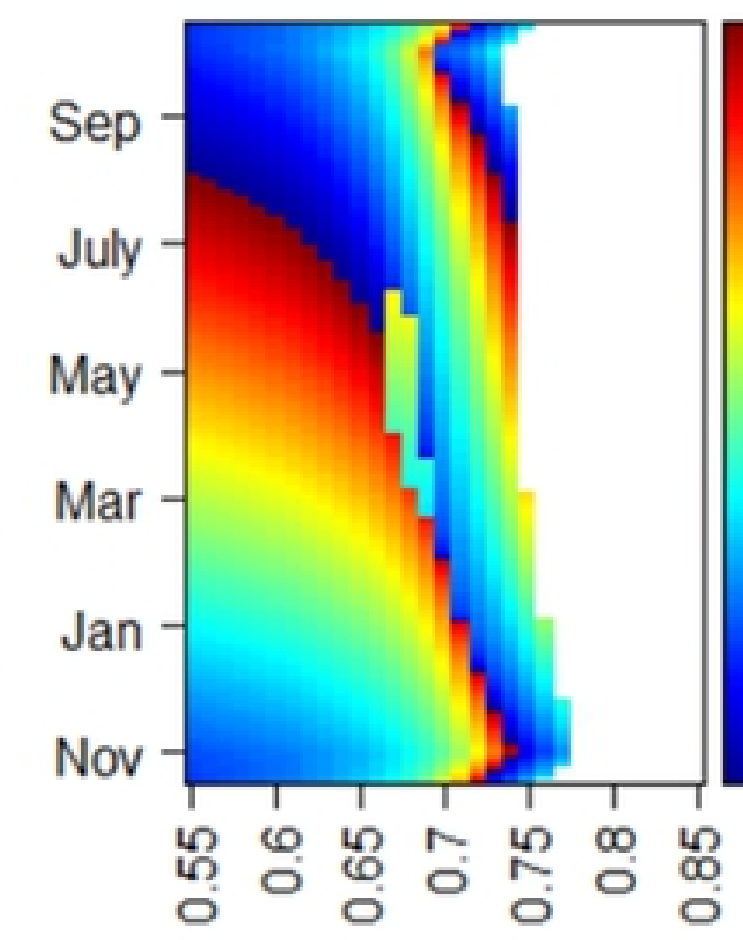

Oct
- Aug
- Jun
- Apr
- Feb
- Dec

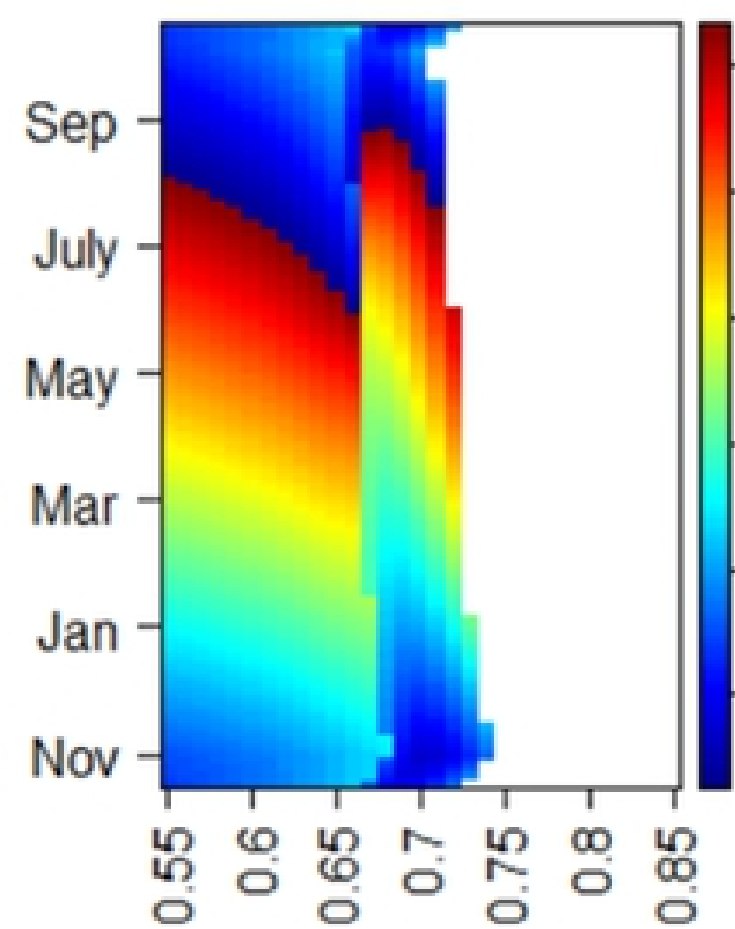

Oct
- Aug
Jun
- Apr
- Feb
Dec

High Birth synchrony

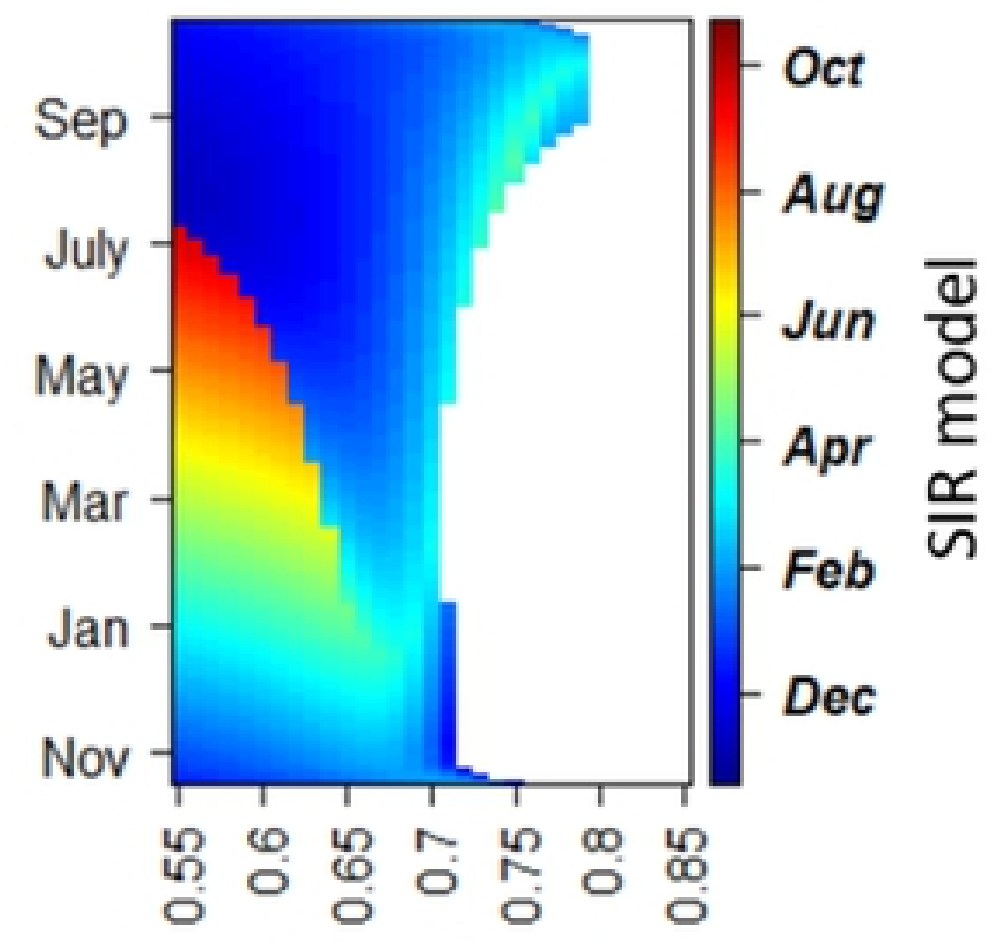

Nov

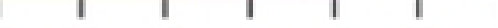

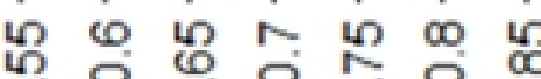

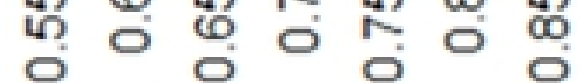

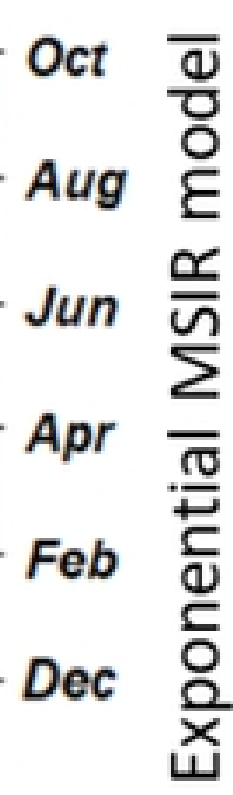

Herd immunity

Fig. 3 
Low Birth synchrony

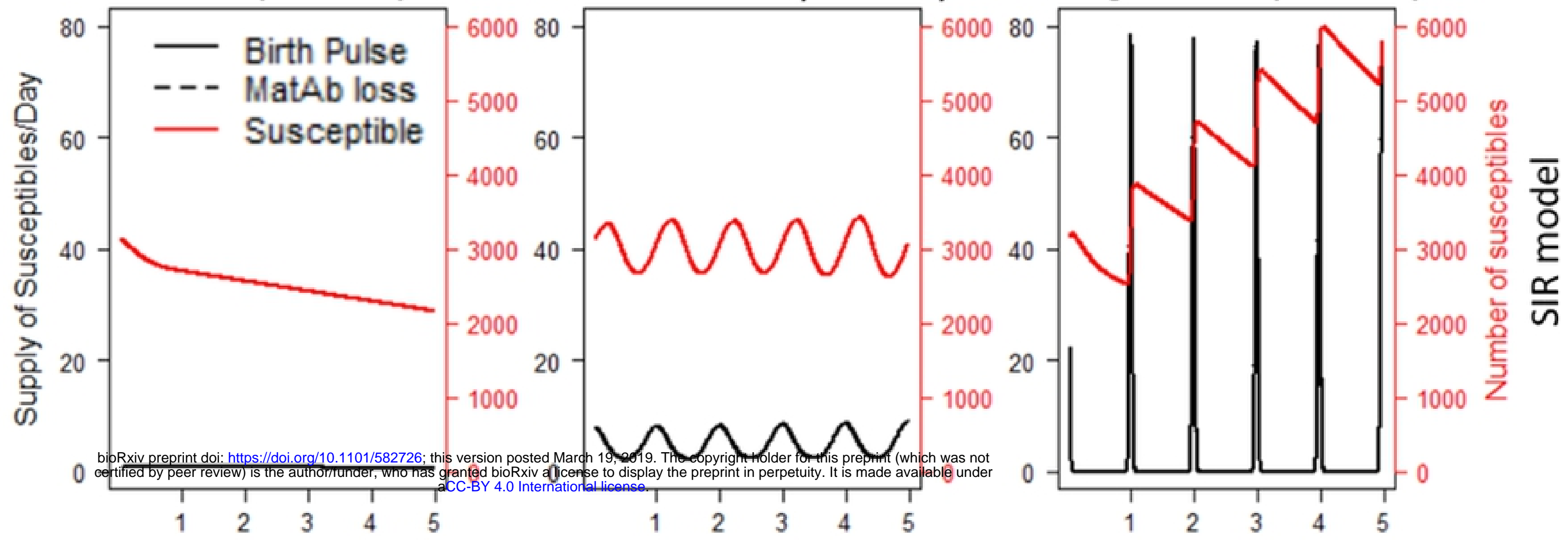

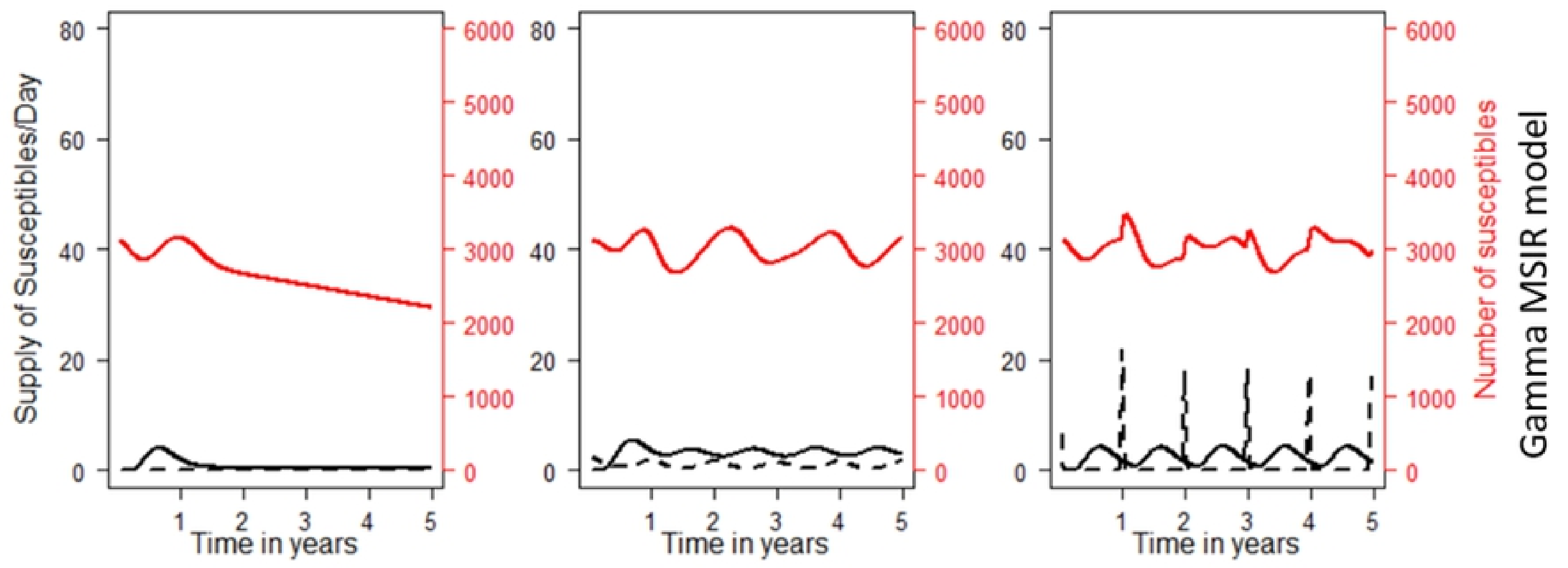

Fig. 4 